\title{
LES COURBES DE LACTATION : LEUR INTÉRET EN ÉLEVAGE (1)
}

par

\section{A. M. LEROY, H. HEIM de BALSAC, J. DELAGE et J. POLY}

L'aptitude laitière des bovins est généralement caractérisée par leur production totale, par lactation, ou par celle obtenue au cours d'une période de référence de 300 ou 330 jours. Cette méthode ne traduit pas l'évolution des productions journalières, qui présente un réel intérêt zootechnique. Il est commode, dans la pratique, de figurer cette évolution sous la forme d'une courbe de lactation, construite à partir des productions journalières, effectivement observées, ou, comme dans les Syndicats de Contrôle Laitier, à l'aide de leur équivalent énergétique exprimé en kilogrammes de lait à $4 \%$ (W. L. GAINES et F. A. DAVIDSON).

Les courbes de lactation. - On constate que la production laitière évolue au cours d'une lactation, suivant un cycle, qui est de même nature chez toutes les vaches laitières.

Six paramètres sont, selon nous, nécessaires pour caractériser une lactation :

La production totale: $\mathrm{R}$;

La durée de la lactation : D ;

La production journalière maxima: Fm ;

La date à laquelle la production commence à décroître : dm ;

Le rythme de croissance de la production dans la phase ascendante de la lactation, du vêlage à la production maxima ;

La persistance de la lactation, qui traduit le taux de décroissance dans la deuxième phase de la lactation.

Avec les données recueillies dans le cadre des Syndicats de Contrôle Laitier, il est possible d'évaluer, de façon suffisamment précise, sur la base d'une réglementation uniforme, la production globale au cours de la lactation, et la longueur de cette dernière. L'enregistrement mensuel rend possible également une estimation de la persistance, mais ne permet pas, par contre, une analyse suffisamment exacte de la phase ascendante de la lactation; pour une telle étude, des contrôles journaliers ou, au moins, bihebdomadaires, s'avéreraient indispensables.

\section{Phase de décroissance de la lactation:}

a) Les travaux antérieurs. La majeure partie des études, portant

(1) Comptes rendus Acad. Agr, 1953, 39, n०2, 97. 
sur les courbes de lactation, se sont occupées uniquement de cette phase, et bien des expressions ont été proposées pour définir la persistance des lactations : "shape figure" de SANDERs (1930), indice $G$ de Fredericksen et OstergaArD (1931), indice P de Nielsen (1938), méthode de Petersen et Collaborateurs (1943), pour n'en citer que quelques-uns.

En fait, peu de travaux de nature mathématique ont été effectués dans ce domaine. La majeure partie des auteurs se contentent, à la suite des publications de BRODY et Collaborateurs (1923) et Gaines (1927) et Collaborateurs (1926) d'admettre une décroissance exponentielle de la production laitière, de la forme $y=\mathrm{Ae}-$ $k t$, où $y$ représente la production laitière en kilogrammes et $t$ le temps en jours à partir du vêlage; le coefficient $k$ comme l'indique GaInes est un indice de persistance. Dans des études ultérieures du même genre, Pontecorvo (1940) propose un coefficient de persistance $\mathrm{P}$, égal à $100 e-k$.

BonNIER (1935), pour une partie de la courbe de décroissance de la production, préfère un ajustement linéaire qu'il justifie statistiquement.

b) Notre étude. Il nous a semblé utile de tester différentes hypothèses de régression, avant de définir la meilleure loi mathématique de décroissance de la production laitière.

A cette fin, nous avons utilisé les données du Syndicat de Contrôle Laitier de Seine-et-Marne, dont nous suivons l'enregistrement avec beaucoup de soins. Nous avons retenu 146 lactations, effectuées en 1949 dans les douze meilleures étables du Syndicat, et construit très exactement chaque graphique individuel en situant les différents contrôles à leur date exacte à partir du vêlage. Cette méthode nous a permis d'établir une courbe moyenne de production pour les 146 animaux du $60^{\mathrm{e}}$ jour après le vêlage — où $97 \%$ des vaches avaient dépassé leur maximum de production - au $300^{\mathrm{e}}$ jour. Son examen nous a conduits à diviser notre étude en deux parties distinctes :

- du $60^{\mathrm{e}}$ au $250^{\mathrm{e}}$ jour ;

- du $250^{\mathrm{e}}$ au $300^{\mathrm{e}}$ jour.

Nous avons étudié la forme mathématique de la première phase de décroissance de la production laitière. Différentes hypothèses de régression ont été testées : régression linéaire, régression exponentielle, régression parabolique, régression hyperbolique.

Nous avons ainsi trouvé que si l'ajustement exponentiel et l'ajustement parabolique sont tous deux statistiquement justifiés, l'ajustement parabolique a, avec nos données, la meilleure signification. 
L'équation de régression parabolique que nous avons obtenue est :

$y=97.10^{6} x^{2}-0,07 x+23,64$, où $y$ est la production journalière en kilogramme, $x$ le temps en jours à partir du vêlage.

Nous avons rassemblé, dans le tableau I, les valeurs moyennes observées pour nos 146 animaux, comparativement aux valeurs calculées à partir de notre équation parabolique.

TABLEAU I

ÉVOLUTION DES PRODUGTIONS DU 60 AU 250 JOUR

\begin{tabular}{c|c|c}
\hline \hline $\begin{array}{c}\text { Classes (temps en jours } \\
\text { écoulés depuis le vêlage) }\end{array}$ & $\begin{array}{c}\text { Production moyenne } \\
\text { observée pour les } \\
146 \text { vaches }\end{array}$ & $\begin{array}{c}\text { Production moyenne théori- } \\
\text { que calculée à partir de } \\
\text { l'équation parabolique }\end{array}$ \\
\cline { 2 - 3 } 60 & 19,806 & 19,781 \\
70 & 19,204 & 19,206 \\
80 & 18,635 & 18,650 \\
90 & 18,042 & 18,114 \\
100 & 17,540 & 17,597 \\
110 & 17,138 & 17,100 \\
120 & 16,681 & 16,622 \\
130 & 16,253 & 16,163 \\
140 & 15,773 & 15,724 \\
150 & 15,305 & 15,304 \\
160 & 14,856 & 14,904 \\
170 & 14,471 & 14,523 \\
180 & 14,137 & 14,161 \\
190 & 13,792 & 13,820 \\
200 & 13,494 & 13,497 \\
210 & 13,184 & 13,193 \\
220 & 12,912 & 12,909 \\
230 & 12,684 & 12,645 \\
240 & 12,428 & 12,400 \\
250 & 12,151 & 12,174 \\
& & \\
\hline \hline
\end{tabular}

Dans notre équation, le terme $x$ traduit la décroissance de la production journalière et le terme $x^{2}$ un ralentissement de cette décroissance à un rythme qui croît comme le carré du temps.

$250^{\mathrm{e}}$ au $300^{\mathrm{e}}$ jour, la production diminue plus rapidement ainsi que le montre le tableau II. Mais l'insuffisance des données ne permet pas d'établir une équation de régression.

\section{Influence de la gestation :}

a) Les résultats antérieurs. Un grand nombre de publications 
TABLEAU II

ÉVOLUTION DES PRODUCTIONS DU $250^{\mathrm{e}}$ AU $300^{\mathrm{e}}$ JOUR

\begin{tabular}{c|c}
\hline \hline $\begin{array}{c}\text { Classes (temps en jours écoulés } \\
\text { depuis le vêlage) }\end{array}$ & $\begin{array}{c}\text { Production moyenne observée } \\
\text { pour 146 vaches }\end{array}$ \\
\cline { 2 - 2 } 250 & 12,151 \\
260 & 11,895 \\
270 & 11,599 \\
280 & 11,214 \\
290 & 10,706 \\
300 & 10,148 \\
\hline
\end{tabular}

s'attachent à l'étude de l'influence de la gestation sur la production laitière. En général, les auteurs indiquent une chute nette de la production vers le cinquième mois de gestation (TURNER, RAGSDale, Brody, 1924). Gaines (1926-1927) propose un terme correctif de nature exponentielle, dont l'application commence à la date de la saillie, et dont l'action n'est vraiment sensible que vers le cinquième mois de gestation. D'autres auteurs chiffrent par un pourcentage l'effet dépressif de la gestation sur la production laitière. Enfin, de récentes publications indiquent une action plus précoce de la gestation, qui serait sensible autour du quatrième mois (MORRISON et Collaborateurs, 1950); EDWARDS (1950).

b) Nos résultats. Les vaches, dont nous avons étudié les courbes de lactation, étaient fécondées en moyenne 140 jours après le vêlage et nous avons constaté qu'une brusque chute de la production laitière - que l'on peut imputer à la gestation - apparaissait au $250^{\mathrm{e}}$ jour, soit 110 jours après la saillie.

Par ailleurs, la représentation parabolique de la courbe de lactation entre le $60^{\mathrm{e}}$ et le $250^{\mathrm{e}}$ jour après le vêlage, nous conduit à penser que la gestation dans sa première phase, pendant les quatre premiers mois, n'a pas, comme on l'affirme souvent, une influence néfaste sur la production laitière; il semble, au contraire, que la persistance de la lactation s'accroisse pendant cette période.

Des études sont en cours actuellement, pour expliquer l'ensemble de ces résultats.

Nous devons néanmoins retenir, dès à présent, qu'un septième paramètre est indispensable si l'on veut définir très exactement une lactation; c'est l'intervalle vêlage-saillie.

\section{Intérêt de l'étude des courbes de lactation en élevage :}

a) Contrôle des conditions d'alimentation dàns un élevage. Les courbes de lactation sont un excellent test biologique des conditions 
dans lesquelles les vaches laitières sont entretenues et elles fournissent, des renseignements précieux sur la valeur de l'affouragement, distribué dans un élevage. L'un de nous (1931-1934), dans une précédente communication, montrait comment il était possible de déceler, à l'examen des graphiques de contrôle laitier, des périodes de sous-alimentation. BüNGER (1939) en Allemagne, dans une plus récente publication, insistait d'ailleurs sur le même problème.

Pour les étables de la région parisienne, il existe en général, deux périodes critiques dans l'alimentation des vaches laitières : la fin de l'hiver où les réserves fourragères sont insuffisantes, en quantité et qualité, et le milieu de l'été, marqué très souvent par la sécheresse. Nous avons pu étudier l'évolution des lactations pour des animaux qui n'étaient pas rationnellement alimentés, à ces deux périodes de l'année.

Lors de la délivrance des certificats de lactation par les Syndicats de Contrôle Laitier, on attire d'ailleurs l'attention des éleveurs sur les baisses anormales de production.

b) Existence de plusieurs types de courbes de lactation. C'est un fait bien connu dans la pratique qu'il y a des animaux qui " tiennent leur lait „, alors que d'autres présentent une décroissance très rapide de leur production journalière. Deux animaux peuvent fournir une même production totale au cours de leur lactation, en répartissant différemment leur production journalière, ainsi qu'en témoignent les courbes observées respectivement par EskEDAL (1949), au Danemark et par nous-mêmes en Seine-et-Marne. L'économie de la production de tels animaux est totalement différente.

En effet, il peut être économiquement intéressant pour l'éleveur de répartir sa production d'une façon assez homogène sur l'ensemble de l'année.

D'autre part, il est certain que des vaches, avec de fortes productions initiales, et une faible persistance, sont plus difficiles à alimenter rationnellement, à cause de leur pointe de production au cours de laquelle des déséquilibres alimentaires peuvent se répercuter, non seulement sur la production globale, mais aussi sur la santé de l'animal. Enfin, les besoins en aliments concentrés, non produits sur l'exploitation, sont en général plus importants pour les vaches qui ont atteint des maxima de production élevés (ENGELER, 1949).

Ces constatations font ressortir tout l'intérêt zootechnique d'animaux à courbes de production régulières. Dans la pratique, il est done utile de pouvoir définir simplement et avec suffisamment d'exactitude, la persistance. On peut définir le coefficient mensuel de persistance comme le rapport de la production d'un mois à eelle 
du mois précédent. Selon nous, la moyenne arithmétique des coefficients mensuels de persistance, qui nous fournit un coefficient moyen - calculés à partir de la décroissance de la production jusqu'à la période de chute brutale, due à l'effet de la gestation - nous donne une excellente estimation de la persistance.

Dans le cas de notre étude, le coefficient moyen de persistance était de $0,925 \mathrm{du} 60^{\mathrm{e}}$ au $250^{\mathrm{e}}$ jour ; il s'abaissait à 0,88 après cette période. Le coefficient moyen, déduit de l'équation de régression parabolique, était de 0,925 .

Afin de préciser si ce caractère de persistance est une caractéristique individuelle pouvant être améliorée par sélection (LUDWIG, Petersen, Fitch, 1943), nous nous proposons, dans une étude ultérieure, d'en calculer l'héritabilité. Les résultats de Johansson (1949) semblent indiquer que ce caractère n'est que faiblement héritable.

Il est utile, pour un chercheur travaillant avec des vaches laitières, de savoir quelle représentation théorique il doit adopter pour les courbes de production. En effet, en utilisant la méthode des périodes successives, il est possible de calculer à partir d'une phase pré-expérimentale et post-expérimentale, une équation de régression, dont la comparaison à la courbe de production observée pendant la période expérimentale peut être extrêmement suggestive. Dans le cas d'expériences de courte durée, il semble que la régression linéaire représente de façon suffisamment précise la décroissance de la production.

Mais, lorsque l'on veut connaître de façon exacte la loi de régression, pour serrer de très près l'influence d'un facteur, une analyse statistique plus détaillée s'impose.

Enfin, il nous paraît souhaitable de tenir compte des résultats précédents pour la tenue des fiches de contrôle laitier-beurrier, en notant régulièrement les dates de saillie et en calculant les coefficients mensuels de persistance dans les conditions que nous avons précisées ci-dessus.

L'évolution des productions journalières est donnée dans ce dernier cas par le tableau III, qui correspond à deux vaches $\mathrm{A}$ et $\mathrm{B}$ ayant vêlé en avril, dont la production en 300 jours était d'environ 4.000 kilogrammes, et les productions maximá respectives de 28 kilogrammes et de 19 kilogrammes. L'aire comprise entre la courbe de lactation et les axes de coordonnées est évidemment la même pour les deux types de lactation, mais la persistance est plus élevée pour la courbe correspondant à la vache $\mathrm{B}$.

Pour répondre à d'éventuelles questions, nous ajouterons les précisions suivantes :

La traite mécanique des vaches, dont les lactations ont été étu- 
TABLEAU III

\begin{tabular}{c|c|c}
\hline \hline $\begin{array}{c}\text { No des contrôles } \\
\text { mensuels }\end{array}$ & $\begin{array}{c}\text { Production de A en kilo } \\
\text { grammes (courbe à faible } \\
\text { persistance) }\end{array}$ & $\begin{array}{c}\text { Production de B en kilo- } \\
\text { grammes (courbe à forte } \\
\text { persistance) }\end{array}$ \\
\cline { 2 - 3 } 1 & 28 & 19 \\
2 & 22 & 16,5 \\
3 & 20 & 15,5 \\
4 & 11,5 & 12,5 \\
5 & 12 & 12 \\
6 & 12 & 12,5 \\
7 & 8 & 11 \\
8 & 7,5 & 11,5 \\
9 & 5,5 & 11,5 \\
10 & 3,5 & 9,5 \\
& & \\
\hline \hline
\end{tabular}

diées ici, ne porte que sur $25 \%$ à peine de l'effectif. Les animaux traits par ce procédé ne présentent pas de courbes d'allure différente.

Des diminutions rapides de la production et des taux de persistance faible ne s'observent que si la traite mécanique est mal utilisée ou mal réglée. Des durées de traite trop longues peuvent être, notamment, l'origine de perturbations.

Le mode de traite ne peut influer de façon sensible sur l'allure de la courbe de lactation qui devient la caractéristique biométrique de la vache laitière.

\section{Travait en Coopération}

\section{Conservatoire National des Arts et Métiers \\ Centre de Biologie Agricole \\ et Industrielle \\ Professeurs}

F. et H. Herm de BaLsaC
Institut National Agronomique

\section{Laboratoire de Zootechnie}

Professeur A. M. Leroy

\footnotetext{
Comité Biologia

Station Biologique

de Buré d'Orval (M.-et.-M.)
} 